\title{
Automated analysis of non-mass-enhancing lesions in breast MRI based on morphological, kinetic, and spatio-temporal moments and joint segmentation-motion compensation technique
}

\author{
Sebastian Hoffmann ${ }^{1}$, Jamie D Shutler ${ }^{2}$, Marc Lobbes $^{3}$, Bernhard Burgeth ${ }^{1}$ and Anke Meyer-Bäse ${ }^{4,5^{*}}$
}

\begin{abstract}
Dynamic contrast-enhanced magnetic resonance imaging (DCE-MRI) represents an established method for the detection and diagnosis of breast lesions. While mass-like enhancing lesions can be easily categorized according to the Breast Imaging Reporting and Data System (BI-RADS) MRI lexicon, a majority of diagnostically challenging lesions, the so called non-mass-like enhancing lesions, remain both qualitatively as well as quantitatively difficult to analyze. Thus, the evaluation of kinetic and/or morphological characteristics of non-masses represents a challenging task for an automated analysis and is of crucial importance for advancing current computer-aided diagnosis (CAD) systems. Compared to the well-characterized mass-enhancing lesions, non-masses have no well-defined and blurred tumor borders and a kinetic behavior that is not easily generalizable and thus discriminative for malignant and benign non-masses. To overcome these difficulties and pave the way for novel CAD systems for non-masses, we will evaluate several kinetic and morphological descriptors separately and a novel technique, the Zernike velocity moments, to capture the joint spatio-temporal behavior of these lesions, and additionally consider the impact of non-rigid motion compensation on a correct diagnosis.
\end{abstract}

Keywords: Non-mass-enhancing lesions; Writhe number; Krawtchouk moments; Zernike velocity moments; Kinetics; Classification; Computer-aided diagnosis; Breast magnetic resonance imaging

\section{Introduction}

Breast cancer is one of the leading causes of death among women in the western hemisphere. Contrast-enhanced magnetic resonance imaging (MRI) of the breast was reported to be a highly sensitive method for the detection of invasive breast cancer [1]. Different investigators described that certain dynamic signal intensity (SI) characteristics (rapid and intense contrast enhancement followed by a washout phase) obtained in dynamic studies are a strong indicator for malignancy [2]. Morphological

\footnotetext{
*Correspondence: ameyerbaese@fsu.edu

${ }^{4}$ Department of Scientific Computing, Florida State University, Tallahassee, FL, 32310-4120, USA

${ }^{5}$ Computational Intelligence and Machine Learning Group, University of Regensburg, Regensburg D-93053, Germany

Full list of author information is available at the end of the article
}

criteria have also been identified as valuable diagnostic tools [3]. Recently, combinations of different dynamic and morphological characteristics have been reported [4,5] that can reach diagnostic sensitivities up to $97 \%$ and specificities up to $76.5 \%$. Many of these studies were performed in the preoperative staging of patients with suspicious lesions (Breast Imaging Reporting and Data System (BI-RADS) 4 and 5) including predominantly tumors with an extension greater than $2 \mathrm{~cm}$. In such cases, MRI reaches a very high sensitivity in the detection of invasive breast cancer due to both the typical appearance (ill-defined shape, stellate borders, and rim enhancement) of malignant tumors and characteristic SI time courses of contrast enhancement. Recent clinical research has shown 
that ductal carcinoma in situ (DCIS) with small invasive carcinoma can be adequately visualized in MRI [6] and that MRI provides an accurate estimation of invasive breast cancer tumor size, especially in tumors of $2 \mathrm{~cm}$ or smaller [7].

However, more than $40 \%$ of the false-negative MR diagnosis are associated with non-mass-like enhancing lesions, thus indicating a lower sensitivity of MRI for these cases. It has been shown that double reading achieves a higher sensitivity but is time-consuming, and as an alternative, a computer-assisted system was suggested [8]. The success of computer-aided diagnosis (CAD) in conventional X-ray mammography [9-13] motivates furthermore the research of similar automated diagnosis techniques in breast MRI.

Non-mass-enhancing lesions exhibit a heterogeneous appearance in breast MRI with high variations in kinetic characteristics and typical morphological parameters [14-16] and have a lower reported specificity and sensitivity than mass-enhancing lesions. The diagnosis of non-mass-like enhancement lesions is thus far more challenging. Malignant lesions such as DCIS and invasive lobular cancer (ILC) exhibit a segmental or linear enhancement pattern, and benign lesions such as fibrocystic changes present as well a non-mass-like enhancement [17]. However, a systematic classification of non-masslike enhancing lesions is not in place. A classification of such lesions would be highly beneficial since they may reduce the biopsies' numbers. The morphological parameters with the highest predictive value in nonmass-enhancing lesions were reported in [15] as segmental distribution, clustered ring enhancement, and a clumped internal architecture. Another study has shown that including kinetic data on dynamic contrast-enhanced imaging and diffusion-weighted MRI imaging in addition to morphological characteristics showed a high diagnostic accuracy in the characterization of these lesions [16].

Research initiatives have been focused on automated analysis of mass lesions [18-24], while very few studies investigated the characterization of the morphology and/or enhancement kinetic features of non-mass lesions [17,25-27]. The studies showed a much lower sensitivity and specificity for non-mass-like enhancement lesions compared with masses and suggested the need for more advanced algorithms for the diagnosis of non-mass-like enhancement.

Current CAD systems are specialized for automated detection and diagnosis of mass-enhancing lesions that are well characterized by shape and kinetic descriptors according to the BI-RADS lexicon. To overcome these problems and revolutionize the state of the art of CAD in breast MRI, we need to focus on correctly capturing and analyzing the unique spatio-temporal behavior of non-mass-enhancing lesions.
In this paper, we present novel techniques for establishing the automated diagnosis of non-mass-enhancing lesions and thus improve the quality of breast MRI postprocessing and reduce the number of missed or misinterpreted cases leading to false-negative diagnosis. In an automated step, we will extract both morphological and kinetic features and use them as potential cues for nonstandard lesion detection. Furthermore, we will apply a novel concept - the Zernike velocity moments [28] - as a unique descriptor that captures the simultaneous behavior of the spatio-temporal behavior of these lesions. We will evaluate quantitatively and qualitatively based on automated classification the utility of novel feature extraction approaches to diagnostically challenging lesions in breast MRI.

\section{Materials and methods}

\subsection{Patients}

The database for non-mass-enhancing lesions includes a total of 84 patient images, all female, with non-massenhancing tumors. All patients had a histopathologically confirmed diagnosis from needle aspiration/excision biopsy and surgical removal. Histologic findings were malignant in 61 and benign in 23 lesions.

\subsection{MR imaging}

MRI was performed with a $1.5 \mathrm{~T}$ system (Magnetom Vision, Siemens, Erlangen, Germany) equipped with a dedicated surface coil to enable simultaneous imaging of both breasts for both types of lesions. The patients were placed in a prone position.

Transversal images were acquired with a short TI inversion recovery (STIR) sequence $(\mathrm{TR}=5,600 \mathrm{~ms}$, $\mathrm{TE}=60 \mathrm{~ms}, \mathrm{FA}=90^{\circ}, \mathrm{IT}=150 \mathrm{~ms}$, matrix size $228 \times$ 182 pixels, slice thickness $3 \mathrm{~mm}$ ). Then, a dynamic T1weighted gradient echo sequence (3-D fast low-angle shot sequence) was performed (TR $=4.9 \mathrm{~ms}, \mathrm{TE}=1.83 \mathrm{~ms}$, $\mathrm{FA}=12^{\circ}$ ) in transversal slice orientation with a matrix size of $352 \times 352$ pixels and an effective slice thickness of $1 \mathrm{~mm}$. The dynamic study consisted of five measurements with an interval of $1.4 \mathrm{~min}$. The first frame was acquired before injection of paramagnetic contrast agent (gadopentatate dimeglumine, $0.1 \mathrm{mmol} / \mathrm{kg}$ body weight, Magnevist $^{T M}$, Schering, Berlin, Germany) immediately followed by the four other measurements.

\subsection{Motion compensation and lesion segmentation}

Automatic motion correction represents an important prerequisite to a correct automated small lesion evaluation [29]. Motion artifacts are caused either by the relaxation of the pectoral muscle or involuntary patient motion and invalidate the assumption of same spatial location within the breast of the corresponding voxels in the acquired volumes for assessing lesion enhancement. 

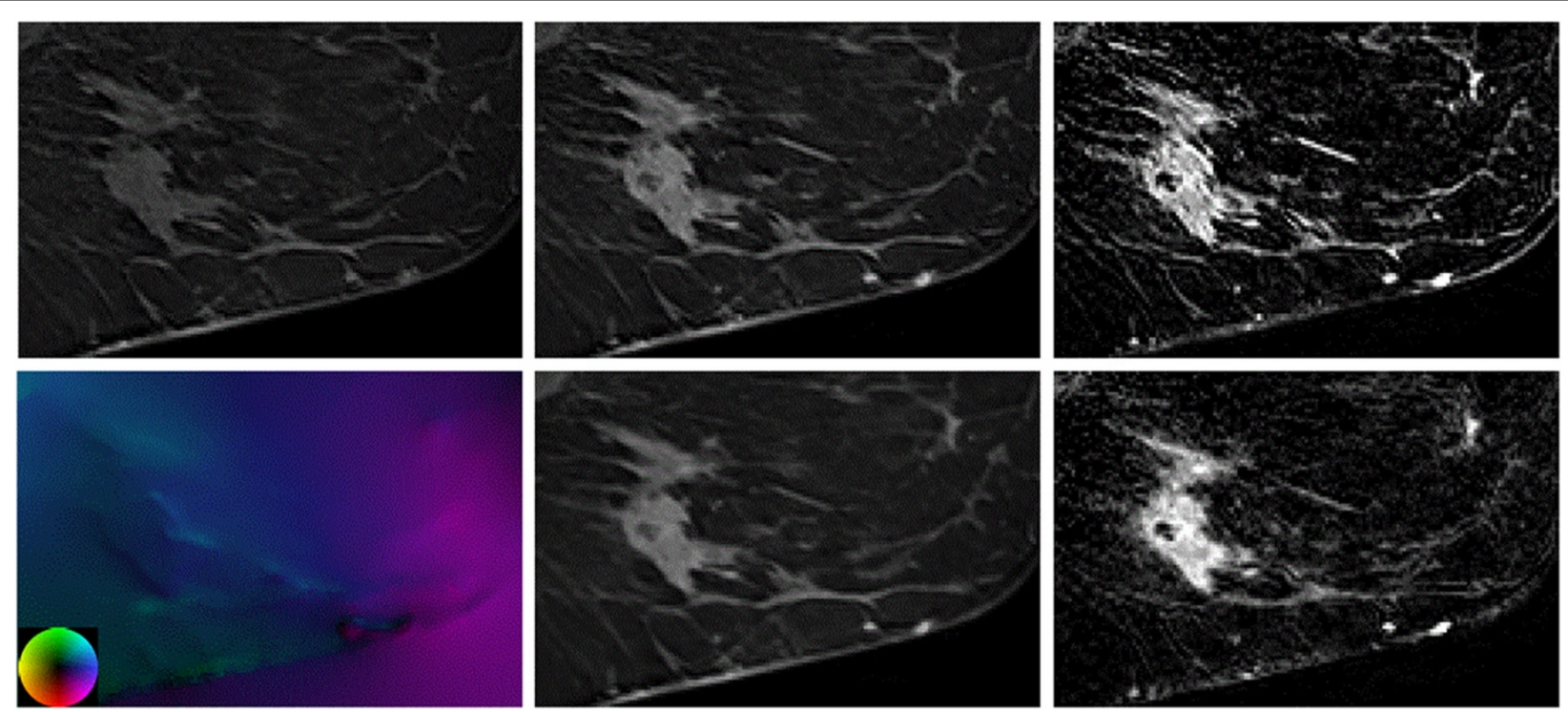

Figure 1 Example results of the motion compensation algorithm. Top left: middle slice of pre-contrast image of a tumor. Top middle: original second post-contrast image. Top right: subtraction image of original images (linearly re-scaled). Bottom left: computed flow field (color code in lower left corner). Bottom middle: motion-compensated post-contrast image. Bottom right: subtraction image using the motion-compensated image (linearly re-scaled).

We employ a motion compensation algorithm based on the technique described in [30] and use separate robustification in the data term. It has been shown that this method yields very good results as it is robust against noise, which is very important for our purposes.

We show in Figure 1 an example of how the motion compensation algorithm works. The flow field between the pre- and post-contrast image is shown in color code, i.e., the color represents the direction of the motion field at a specific location, whereas the brightness corresponds to the magnitude. Note that the three-dimensional image is represented by the middle transverse slice as usual. Furthermore, the motion-compensated post-contrast image is visualized. Although the magnitude of the depicted displacement field is relatively small, it is still very useful to remove small artifacts in the subtraction image. Due to the motion compensation, the images are more aligned with each other, and thus, the boundary of the breast becomes also less visible since it is not enhanced. Similar improvements can also be observed in the tumor itself and the surrounding tissue. Note that the images as well as the flow consist of three dimensions. This means that the algorithm tries to find the best possible displacement in all three directions. This is the reason why the structures in the original and the motion-compensated image can look differently when only considering one slice, because parts from different slices, not visible in the example, can have an influence on the depicted slice and vice versa.

Tumor segmentation represents the correct identification of the spatial location of a tumor. Manual segmentation performed by a radiologist is considered the gold standard. However, expert segmentation is not highly precise and prone to inter-observer and intra-observer variability, and it might include also non-enhancing tissue. It is time-consuming by viewing both spatial and temporal profiles and thus examining many series of enhanced data and profiles of pixels while determining the lesion boundary. To overcome these problems, we employ as an automatic segmentation method an active contour segmentation without edges as proposed by Chan and Vese [31] and improve the algorithm by taking into account the three-dimensional image sequence and by adding a smoothness assumption to level set function:

Using finite difference methods (FDM) and solving the following Hamilton-Jacobi equation (Euler Lagrange equation corresponding to active contour without edges) give the boundary of target with the given initial level set function $\phi_{0}(x, y, z)$.

$$
\begin{aligned}
\frac{\partial \phi}{\partial t} & =\delta_{\epsilon}\left[\mu \nabla \cdot\left(\frac{\nabla \phi}{|\nabla \phi|}\right)-\lambda_{1} \frac{1}{n} \sum_{d=1}^{n} \gamma_{d}\left(f_{d}-c_{d}^{\text {in }}\right)^{2}+\lambda_{2} \frac{1}{n} \sum_{d=1}^{n} \gamma_{d}\left(f_{d}-c_{d}^{\text {out }}\right)^{2}\right]+\theta \nabla \cdot\left(\frac{\nabla \phi}{|\nabla \phi|}\right)=0 & & \text { in }(0, \infty) \times \Omega \\
\phi(0, x, y, z) & =\phi_{0}(x, y, z) & & \text { in } \Omega \\
\frac{\delta_{\epsilon}(\phi)}{|\nabla \phi|} \frac{\partial \phi}{\partial \mathbf{n}} & =0 & & \text { on } \partial \Omega
\end{aligned}
$$


where $\left\{f_{d}\right\}_{d=1, . ., n}$ is the image sequence for a given case consisting of $n$ three-dimensional images. In our case, $n=5 . c^{\text {in }}=\left(c_{1}^{\text {in }}, \ldots, c_{n}^{\text {in }}\right)^{T}$ are the average gray values for each image for the inside region, and similarly, $c^{\text {out }}=\left(c_{1}^{\text {out }}, \ldots, c_{n}^{\text {out }}\right)$ for the outside region. Since the contrast agent has a certain in-take-time, the consecutive images will be weighted more. The weighting factors $\gamma_{1}, \ldots, \gamma_{n} \geq 0$ take into consideration accordingly this information content of an image. We also use $\delta_{\epsilon}(x):=$ $\frac{1}{2}\left(1+\frac{2}{\pi} \arctan \left(\frac{x}{\epsilon}\right)\right), \mathbf{n}:=$ outer normal direction of $\partial \Omega$, and scalar parameters $\mu, \lambda_{1}, \lambda_{2}$.

Let $\phi^{N}(x, y, z)$ be the $N$ th step evolution of $\phi(t, x, y, z)$ in FDM. Then, the curve $\gamma$ for detected boundary is defined

$$
\begin{aligned}
\gamma & :=\left\{(x, y, z) \subset \mathbb{R}^{3}: \phi^{N}(x, y, z)\right. \\
& \left.=0 \text { for }\left\|\phi^{N}(x, y, z)-\phi^{N-1}(x, y, z)\right\|<\tau\right\}
\end{aligned}
$$

where $\tau$ is the tolerance. To get a good approximation of a global optimizer of this non-convex problem, we make use of a coarse-to-fine multigrid implementation of the algorithm. The parameter $\eta \in[0.5,1)$ thereby determines the downsampling factor.

For the numerical simulation, we choose the following parameters: $\lambda_{1}=1, \lambda_{2}=1,\left(\gamma_{1}, \gamma_{2}, \gamma_{3}, \gamma_{4}\right)^{T}=(1,3,2,1)^{T}$, $\eta=0.8$, and $\theta=0.1$. An example of the proposed segmentation method can be seen in Figure 2.

\section{Enhancement kinetic features}

While mass-enhancing lesions exhibit a typical kinetic behavior that is distinctive for malignant and benign lesions, non-mass-enhancing lesions have kinetic characteristics that are far less well characterized and of limited accuracy in discriminating between malignant and benign behavior [26]. As a dynamical feature, the slope of the relative signal intensity enhancement (RSIE) is used in most current CAD systems. Very few studies exist for analyzing the kinetics of non-masses.

Besides the description of the texture, we also want to characterize the enhancement of the tumor due to the contrast agent. We are focusing on this in the following mainly by considering the mean gray values of each tumor region and how it develops over time. Therefore, we consider trivial facts like the magnitude of the enhancement as well as more sophisticated methods like the Fourier transform or regression methods. All these approaches are described in the following.

\subsection{Slope of mean values}

A simple but important feature is the slope of the enhancement. For a given image $f$, we consider the mean value inside the tumor region:

$$
\mu=\frac{1}{|T|} \sum_{\boldsymbol{p} \in T} f_{\boldsymbol{p}}
$$

Here, $\boldsymbol{p} \in T$ represent the pixels within a tumor region $T$. Computing the mean for each of the $I$ images belonging to one tumor yields the mean values $\mu_{1}, . ., \mu_{I}$, from which we consider the difference between subsequent values

$$
d_{i}=\frac{\mu_{i+1}-\mu_{i}}{\Delta t} \quad i=1, \ldots, I-1
$$

where $\Delta t$ denotes the time between the images. We take these values as additional features. This is the same as computing the average of all slopes between single pixels.
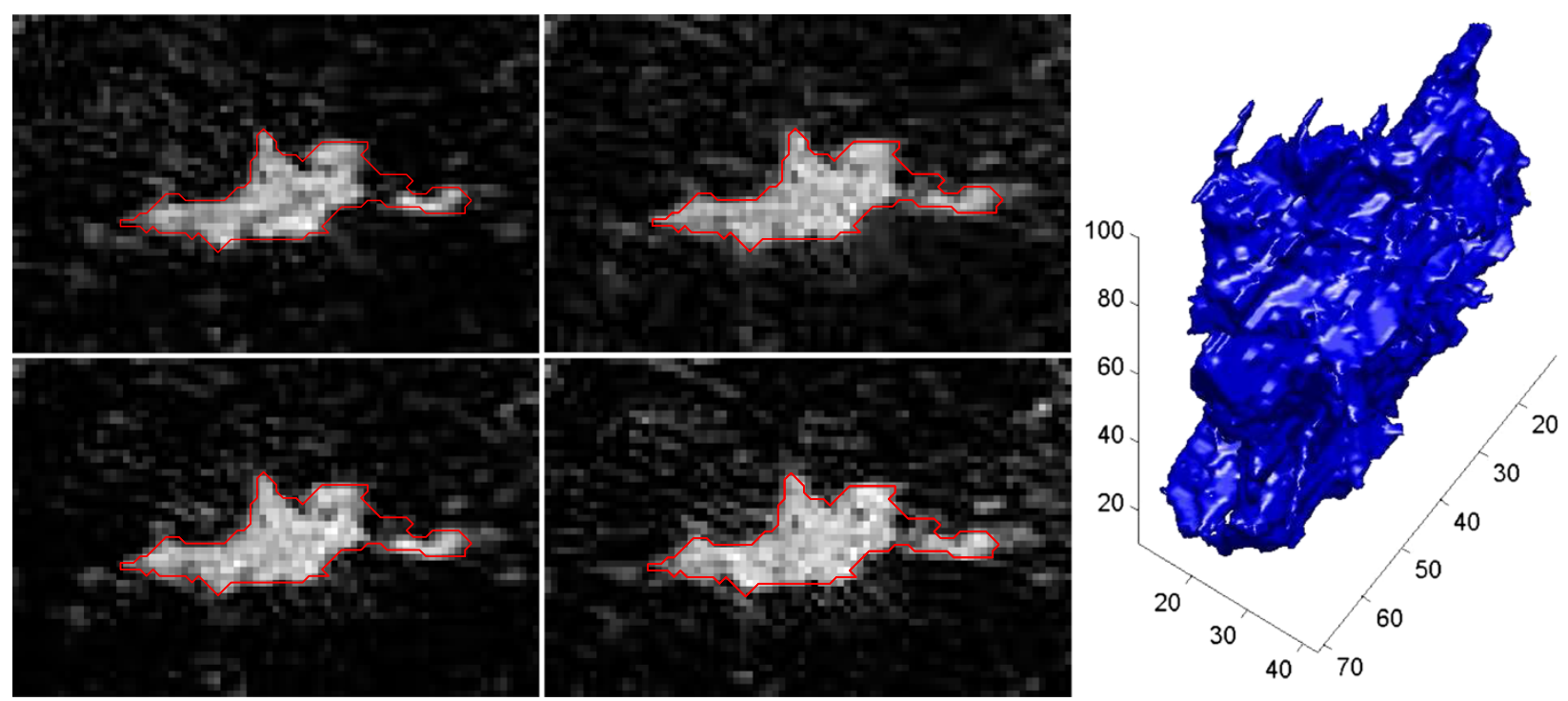

Figure 2 Segmentation method example. Left: four subtraction images of the respective post-contrast and the pre-contrast image. The result of the segmentation is shown as a red line. $(\mu=300.0, \alpha=5.0)$ Right: $3-D$ view of the segmented tumors. 


\subsection{Regression methods}

In order to get better descriptors of the enhancement, we are now considering different classes of functions and perform regression, i.e., we try to find parameters of the given model which approximate the given points best. Instead of just considering the mean values, we consider the mean values of the post-contrast images in relation to the pre-contrast image. We therefore define the relative enhancement (RE) as

$$
s_{i}=\frac{\mu_{i}-\mu_{1}}{\mu_{1}} \quad i=1, \ldots, I
$$

Given the points $\left\{\left(t_{i}, s_{i}\right)^{\top}\right\}_{i=1, \ldots, I}$ with $t_{i}=i \Delta t$, we to try apply regression with different functions described below.

\subsubsection{Linear function}

This feature is also known as the relative signal intensity enhancement and was already used by Retter and Steinbrücker for mass-like tumors [32-34]. The idea behind it is that in the beginning, both benign and malignant tumors have the same behavior by absorbing a lot of the contrast agent leading to a rapid enhancement. However, the temporal behavior can be highly diagnostic for the type of the tumor. While benign mass tumors tend to have a further increase of the enhancement, malignant tumors are prone to have a rapid washout effect. The question is if this idea can be transferred to the nonmass-like tumors. Jansen et al. found out in their experiments that this criteria is not valid anymore in this case [35]. However, we want to examine if it is also the case for our data set, and therefore, we are incorporating this feature in the classification process, too.

The idea is to approximate the last three values of the relative enhancement by a linear function $g(t)=a t+b$ in order to describe the enhancement behavior towards the end. We set $I=5$ as it is the case in our data set. The parameters $a$ and $b$ have to be optimized in order to fit the given values. We are, however, only interested in the ascend of the function $a$. The optimal value can be computed as the solution of the following least squares regression:

$$
\underset{a, b}{\operatorname{argmin}}\|\underbrace{\left(\begin{array}{cc}
t_{3} & 1 \\
\vdots & \vdots \\
t_{I} & 1
\end{array}\right)}_{=: A}\left(\begin{array}{c}
a \\
b
\end{array}\right)-\underbrace{\left(\begin{array}{c}
s_{3} \\
\vdots \\
s_{I}
\end{array}\right)}_{=: y}\|
$$

which yields as solution

$$
\left(\begin{array}{l}
a \\
b
\end{array}\right)=\left(A^{\top} A\right)^{-1} A^{\top} y
$$

After some computations, we get for $a$ the following result which is used as a feature:

$$
a=\frac{\sum_{i=3}^{I} t_{i} \sum_{i=3}^{I} s_{i}-I \sum_{i=3}^{I}\left(t_{i} s_{i}\right)}{\left(\sum_{i=3}^{I} t_{i}\right)^{2}-I \sum_{i=3}^{I}\left(t_{i}\right)^{2}}
$$

\subsubsection{Exponential function}

Exponential functions have the potential to fit the points of the relative enhancement better and are therefore used in several papers. Jansen et al. [35], for example, used the following function

$$
g(t)=A \cdot\left(1-e^{-\alpha(t-1)}\right) \cdot e^{-\beta(t-1)}
$$

with the parameters $A, \alpha$, and $\beta$. An example of the fitted function can be seen in Figure 3. It is now possible to fit the values instead of approximating them as it is the case with linear functions. The parameters of the nonlinear fitting function can be gained using an iterative algorithm. We used the Nelder-Mead method [36], which is already implemented in Matlab ${ }^{\odot}$. Having these values, Jansen et al. proposed to compute some properties based on the function as features:

- Initial area under the curve until a specified time $\tau$. We use $\tau=3 \Delta t$ for our experiments.

$$
\mathrm{iAUC}=A \cdot\left(\frac{1-e^{-\beta \tau}}{\beta}+\frac{e^{-(\alpha+\beta) \tau}-1}{\alpha+\beta}\right)
$$

- Initial slope

$$
\text { iSlope }=A \alpha
$$

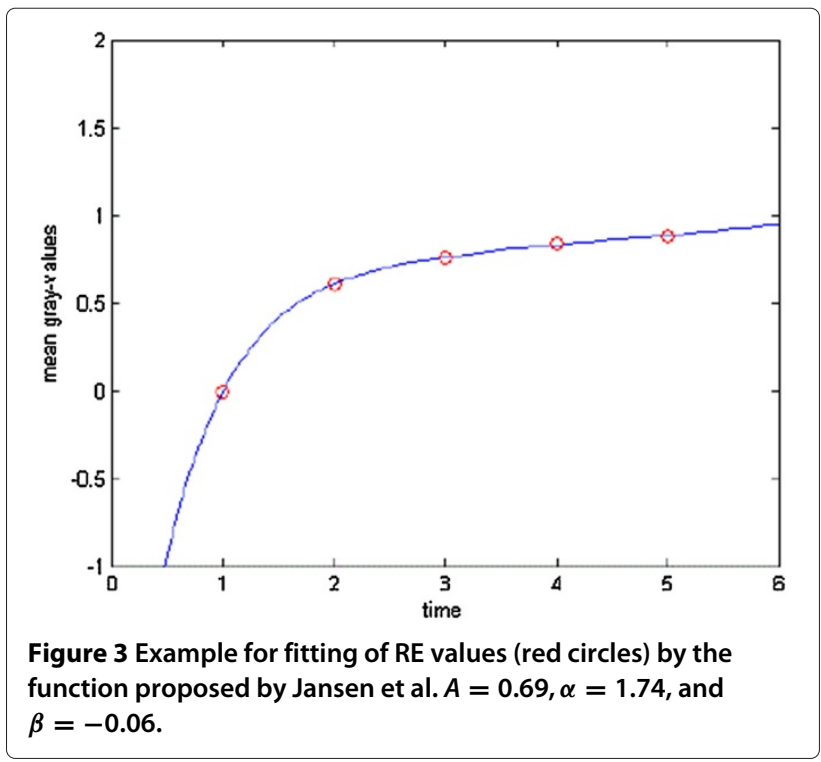


-

Time to peak enhancement

$$
\mathrm{T}_{\text {peak }}=\frac{1}{\alpha \log \left(1+\frac{\alpha}{\beta}\right)}
$$

- Curvature at the peak

$$
\kappa_{\text {peak }}=-A \alpha \beta
$$

In contrast to the original paper of Jansen et al., we also include the parameters $A, \alpha$, and $\beta$ in the feature list.

\section{Morphological features}

Morphological characteristics contain valuable information about a lesion's type. Combined with kinetic properties, one could expect a higher accuracy. Furthermore, non-mass-enhancing lesions such as DCIS or ICS can be better differentiated based on morphological properties [25]. In a previous work [37], we have considered features that describe the geometric characteristics of the shape and local moments such as Krawtchouk to identify the non-smooth surface.

In this section, we will focus on features that are solely based on the morphology of the tumor, i.e., we will study the geometric structure without considering the gray values. As a result of the segmentation algorithm, we have a binary image telling us which pixels belong to the tumor and which do not. This representation, however, is not sufficient for our needs. A better representation would be a triangulation of the surface points. This is done using the built-in method in Matlab. It uses an edge-based algorithm and returns for a given binary tumor image a set of vertices $V$ consisting of 3-D points and faces $F$ defining the triangles connecting the vertices in $V$. Based on this representation, we can compute the following features.

\subsection{Writhe number}

The writhe number describes to which extent a surface is twisted and coiled in itself. It has been introduced by Fuller for the description of the writhing of curves in space [38]. Lauric et al. used this idea and transferred it to surfaces $[39,40]$. Their application to the analysis of intracranial aneurysms yielded promising results. The Writhe number is computed for vertices along the surface of the tumor and represents geometrically the surface asymmetries. Additionally, it has an interesting physical interpretation: it describes the 'twisting force' acting on an object. The writhe number quantifies the twisting force that acts at each point on the surface of a lesion. Given a surface $S$, they defined a relationship $w$ between two different points $\boldsymbol{p}, \boldsymbol{p}^{\prime} \in S$ as

$$
w\left(\boldsymbol{p}, \boldsymbol{p}^{\prime}\right)=\frac{\left[\boldsymbol{n}_{\boldsymbol{p}}, \boldsymbol{p}^{\prime}-\boldsymbol{p}, \boldsymbol{n}_{\boldsymbol{p}}^{\prime}\right]}{\left\|\boldsymbol{n}_{\boldsymbol{p}}\right\| \cdot\left\|\boldsymbol{p}^{\prime}-\boldsymbol{p}\right\| \cdot\left\|\boldsymbol{n}_{\boldsymbol{p}}^{\prime}\right\|}
$$

where $\boldsymbol{n}_{\boldsymbol{p}}, \boldsymbol{n}_{\boldsymbol{p}}^{\prime}$ denote the surface normals at the points $\boldsymbol{p}$ and $\boldsymbol{p}^{\prime}$ and $[\boldsymbol{a}, \boldsymbol{b}, \boldsymbol{c}]:=\boldsymbol{a}^{\top}(\boldsymbol{b} \times \boldsymbol{c})$. Thereby, $\boldsymbol{b} \times \boldsymbol{c}$ denotes the cross product of the vectors $\boldsymbol{b}$ and $\boldsymbol{c}$. The writhe number of a point $\boldsymbol{p} \in S$ is then given as

$$
W(\boldsymbol{p})=\int_{\boldsymbol{p}^{\prime} \in S \backslash\{\boldsymbol{p}\}} w\left(\boldsymbol{p}, \boldsymbol{p}^{\prime}\right) \mathrm{d} S
$$

The discrete counterpart is

$$
W(\boldsymbol{p})=\sum_{\boldsymbol{p}^{\prime} \in S \backslash\{\boldsymbol{p}\}} w\left(\boldsymbol{p}, \boldsymbol{p}^{\prime}\right) \Delta \boldsymbol{p}^{\prime}
$$

The value $\Delta \boldsymbol{p}^{\prime}$ is the area assigned to the point $\boldsymbol{p}^{\prime}$.

We now want to compute the writhing number for the vertices in $S=V$. Let a face in the set $F$ be given by the three vertices $\boldsymbol{v}_{\mathbf{1}}, \boldsymbol{v}_{\mathbf{2}}, \boldsymbol{v}_{\mathbf{3}} \in V$. We can then compute the area $A$ and normal $\boldsymbol{n}$ for this face as

$$
n=\left(v_{2}-v_{1}\right) \times\left(v_{3}-v_{1}\right), \quad A=\frac{1}{2}\|n\|
$$

The normal of each vertex is then computed by averaging the normals of the faces containing the vertex. Similarly, to obtain a measurement of the value $\Delta p$ of a vertex $\boldsymbol{p}$, the areas of the faces containg $\boldsymbol{p}$ are averaged. Having these values, we can compute the writhe number for every vertex. Finally, we compute the first-order statistics of the values.

\subsection{Krawtchouk moments}

Krawtchouk moments represent a set of orthonormal polynomials associated with the binomial distribution. Their mathematical derivation is exemplarily described in [41].

Weighted 3-D Krawtchouk moments [42] have several advantages compared to other known methods: (1) they are defined in the discrete field and thus do not introduce any discretization error like spherical harmonics defined in a continuous field, and (2) low-order moments can capture abrupt changes in the shape of an object. These moments form a very compact descriptor of a tumor, achieved in a computationally efficient way. In our previous work [43], we have extended them to capture the three-dimensional properties of a shape. The resulting moments are given by

$$
\begin{aligned}
R_{n k l m}= & \frac{1}{v+2 \gamma} \sum_{r=0}^{u-1} \sum_{s=0}^{v-1} \sum_{t=0}^{\sigma-1} \sum_{u=0}^{\xi-1}\left(K_{n}(r ; p, N-1)\right. \\
& \times \exp \left(-i \frac{2 \pi s k}{v}\right) \exp \left(-i \frac{2 \pi t l}{\sigma}\right) \exp \left(-i \frac{2 \pi u m}{\xi}\right) \\
& \left.\times f\left(r, \frac{2 \pi s}{v}, \frac{\pi t+0.5}{\sigma}, \frac{2 \pi u}{\xi}\right)\right)
\end{aligned}
$$


where the image $f$ is represented in the radial polar system. To make them rotationally invariant, one considers the norm of the moments. The function $K_{n}$ refers to the Krawtchouk polynomial of order $n$. The notations and detailed mathematical derivations are described in [43].

\subsection{Zernike velocity moments}

Velocity moments (Zernike or Cartesian) are based around the statistical center of mass (COM) of an image and are primarily designed to describe a moving and/or changing shape in an image sequence. The method enables the structure of a moving shape to be described, together with any associated motion information, and so they are termed spatio-temporal moments. They are formulated as a weighted sum of moments over a sequence of frames of length $\mathrm{T}$, where the weight factor is a real-valued scalar function of the displacement of the COM between consecutive frames. Zernike velocity moments are based on orthogonal Zernike polynomials, and so the moments are less correlated and require a lower precision for their calculation in comparison to their Cartesian counterparts. Zernike velocity moments are defined as

$$
A_{m n \alpha \gamma}=\frac{m+1}{\pi} \sum_{i=2}^{I} \sum_{x} \sum_{y} U(i, \alpha, \gamma) S(m, n) P_{i_{x y}}
$$

where $P_{i_{x y}}$ is the pixel at location $x, y$ of the $i$ th image in the sequence. They are bounded so that $\left(x^{2}+y^{2}\right) \leq 1$, while the shape's structure contributes through the orthogonal complex Zernike polynomials [44]

$$
S(m, n)=\left[V_{m n}(r, \theta)\right]^{*}
$$

and velocity is introduced using the COM [44]:

$$
U(i, \alpha, \gamma)=\left(\overline{x_{i}}-\overline{x_{i-1}}\right)^{\alpha}\left(\overline{y_{i}}-\overline{y_{i-1}}\right)^{\gamma}
$$

where $\overline{x_{i}}$ is the current COM in the $x$ direction, while $\overline{x_{i-1}}$ is the previous COM in the $x$ direction, and $\overline{y_{i}}$ and $\overline{y_{i-1}}$ are the equivalent values for the $y$ direction. Velocity moments have been previously applied to analyze human motion [28]. Here we use the Zernike velocity moments to describe the breast MRI image sequences. These spatiotemporal moments (or descriptors) allow us to capture the spatial variation of contrast enhancement over time within the image sequence (i.e., they capture the changes in signal intensity and shape between scans).

\section{Classification techniques}

The following section gives a description of classification methods applied to evaluate the effect of automated classification of diagnostically challenging breast MRI lesions such as foci and non-mass-enhancing lesions based on several feature extraction methods.
Let us assume that $\mathbf{x}$ describes a K-dimensional feature vector and that there are $J$ classes and $N_{j}$ samples available in group $j$. The mean in group $j$ is given by $\mu_{j}$, and the covariance matrix is given by $\Sigma_{j}$.

As classification techniques, we employ support vector machines (SVM) with different kernels.

\subsection{Support vector machines}

SVMs represent an important technique for lesion classification in medical imaging. The key point of this technique is to determine a hyperplane $H=a v+b$ that separates the feature vectors $\vec{v}_{i}, i=1, \ldots, n$ in their $d$ dimensional domain in two classes $\vec{v}_{i} \in\{M, B\}$. First, let us assume that our data set is linearly separable and that we can find a pair $(\vec{w}, b)$ that fulfills

$$
\begin{array}{ll}
\vec{w}^{T} \vec{v}_{i}+b \geq+1 & \vec{v}_{i} \in M \\
\vec{w}^{T} \vec{v}_{i}+b \leq-1 & \vec{v}_{i} \in B
\end{array}
$$

Furthermore, we want both inequalities to be sharp. The best hyperplane is the one that maximizes the distance (margin) of the two parallel hyperplanes defined in (5). Since the distance of a hyperplane to the origin is $\frac{b}{\|\vec{w}\|}$, we want to maximize $\frac{2}{\|\vec{w}\|}$. This leads to the following constrained optimization problem: Find $(\vec{w}, b)$ so that $L(\vec{w})=\frac{\|\vec{w}\|}{2}$ is minimal subject to the condition

$$
f\left(\vec{v}_{i}\right)= \begin{cases}+1 & \text { if } \vec{v}_{i} \in M \\ -1 & \text { if } \vec{v}_{i} \in B\end{cases}
$$

Of course this only works if all the variables are linearly separable, which cannot be assumed. This problem is solved by introducing positive slack variables $\theta_{i}, i=$ $1, \ldots, n$ which leads us to a minimization of $L(\vec{w})=\frac{\|\vec{w}\|}{2}+$ $\alpha \sum_{i=1}^{n} \theta_{i}$ subject to

$$
f\left(\vec{v}_{i}\right)=\left\{\begin{array}{l}
+1, \text { if } \vec{w}^{T} \vec{v}_{i}+b>1-\theta_{i} \\
-1, \text { if } \vec{w}^{T} \vec{v}_{i}+b<-1+\theta_{i}
\end{array}\right.
$$

An additional challenge appears if there is a non-linear function that separates the variable, since the common approach would fail under these circumstances. Aizerman et al. refined this method by using non-linear kernel functions instead of the scalar product which maps the variables onto another space [45]. The optimal hyperplane computed corresponds to a non-linear function in the original feature space.

\section{Experimental results}

We analyze both quantitatively and qualitatively the effect of the previously introduced features for nonmass-enhancing lesions in a computer-aided diagnosis system. We choose as a classifier a SVM with different kernels as described in Table 1. The area under the 
Table 1 Classifiers employed for lesion classification

\begin{tabular}{ll}
\hline Name & Description \\
\hline SVM kernel 1 & SVM classification with a linear kernel \\
SVM kernel 2 & SVM classification with a polynomial kernel \\
SVM kernel 3 & SVM classification with radial basis kernel \\
SVM kernel 4 & SVM classification with sigmoidal kernel \\
\hline
\end{tabular}

ROC curve (AUC) will serve as a quantitative evaluation measure for the proposed CAD system.

Figure 4 gives an overview of the classification results for both motion-compensated data (blue bars) as well as uncompensated data (red bars). The most important fact is that motion compensation improves in most cases the AUCs, suggesting that motion artifacts play an important role in correct diagnosis of non-mass-enhancing lesions. The dynamical features such as parameters extracted from the approximation of the RSIE curves (see Figure 4a) yield the best results, while morphological features like the Writhe number (see Figure 4e) are close to the dynamical features. The averaged Zernike descriptors provide a lower classification rate; however, it is higher than the slope of the mean values as shown in Figure 4d.

A recent study for mass-like-enhancing lesions [5] has shown that both morphological and kinetic features outperform spatiotemporal features. The morphological features were the most discriminative, suggesting that the lesion's morphology - in concordance with clinical practice - determines the further evaluation based on a needle biopsy. Kinetic features, on the other hand, seem to be more discriminative in the case of non-mass-like enhancing lesions followed by the morphological ones.

\section{Conclusion}

We have implemented and tested the most comprehensive computer-aided diagnosis system for non-massenhancing lesions in the literature consisting of motion compensation, segmentation, and feature extraction and classification. These types of lesions are diagnostically challenging since typical kinetic or morphological descriptors are not known so far. The present paper evaluated the discriminative power of a novel joint spatiotemporal technique, the Zernike velocity moments, versus single kinetic or shape descriptors for the diagnosis of these lesions in combination with or without motion compensation. Motion compensation proved in most cases to be diagnostically relevant. The best discriminative features are based on the dynamical properties of an approximation of the RSIE curve, suggesting that kinetics such as wash-in and washout parameters plays a key role in correctly diagnosing non-mass-enhancing lesions and confirms existing studies. The next best feature set is represented by the Writhe number computed for vertices along the surface of the tumor, describing the surface asymmetries. Intuitively, our results for the Writhe

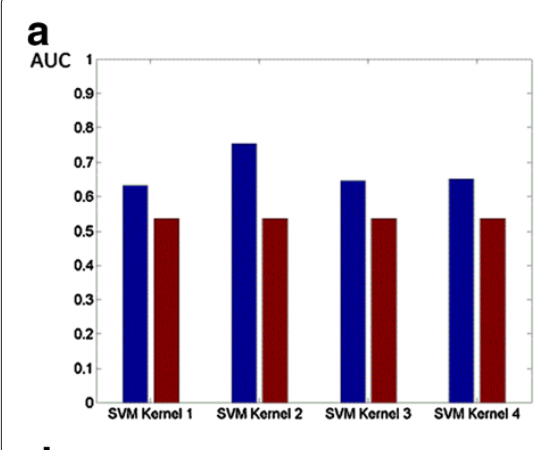

AUC

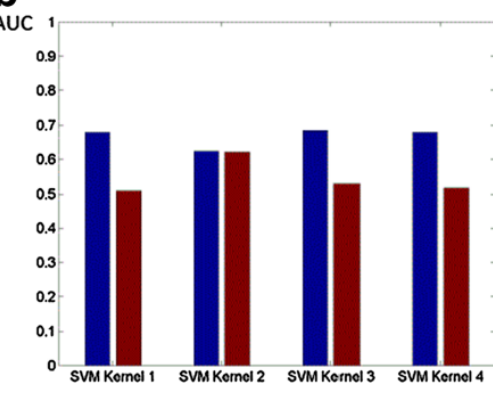

e

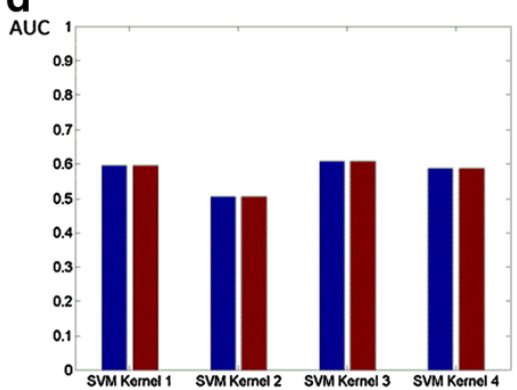

AUC

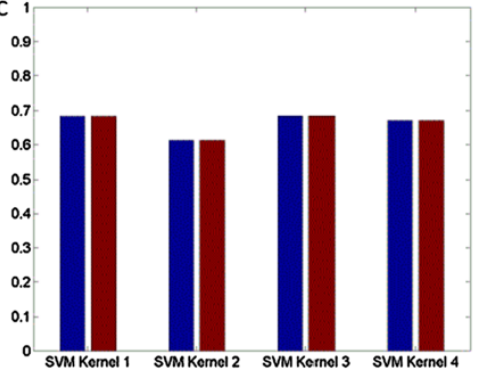

C

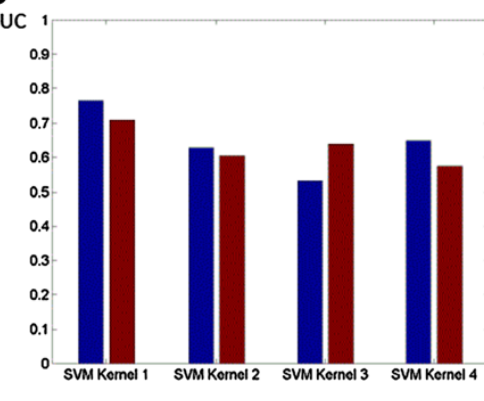

f

AUC

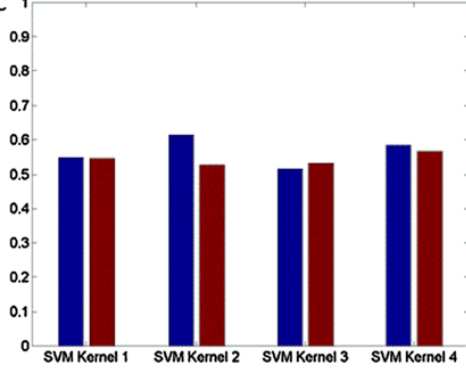

Figure 4 AUCs of SVM applied to kinetic and morphological features, separately. Four different kernels and both motion-compensated (blue bars) as well as original data (red bars) were used to compute the features. (a) Regression with exponential function, (b) Krawtchouk moments, (c) Regression with linear function, (d) Morphology, (e) Writhe number, and (f) Zernike velocity moments. 
number have shown that the more twisted the surface of a lesion is, the greater the likelihood for malignancy is. The spatio-temporal moments, alone, seem to provide an independent characterization of the tumor, and we suspect that in combination with morphological and kinetic features, they will improve their discriminative power. Future studies will be necessary to evaluate in a large trial the effectiveness of these novel descriptors for non-mass-enhancing lesion diagnosis.

\section{Competing interests}

The authors declare that they have no competing interests.

\section{Acknowledgements}

This research was supported in part by NIH grant 5K25CA106799-05.

\section{Author details}

${ }^{1}$ Saarland University, Saarbrücken 66041, Germany. ${ }^{2}$ Plymouth Marine Laboratory, Prospect Place, The Hoe, Plymouth, PL1 3DH, UK. ${ }^{3}$ Maastricht University Medical Center, Maastricht 6202 AZ, Netherlands. ${ }^{4}$ Department of Scientific Computing, Florida State University, Tallahassee, FL, 32310-4120, USA. ${ }^{5}$ Computational Intelligence and Machine Learning Group, University of Regensburg, Regensburg D-93053, Germany.

Received: 15 February 2013 Accepted: 24 July 2013

Published: 15 November 2013

\section{References}

1. S Orel, MD Schnall, CM Powell, MG Hochman, LJ Solin, BL Fowble, MH Torosian, EF Rosato, Staging of suspected breast-cancer-effect of MR imaging and MR-guided imaging and biopsy. Radiology. 196, 115-122 (1995)

2. CK Kuhl, P Mielcareck, S Klaschik, C Leutner, E Wardelmann, J Gieseke, H Schild, Dynamic breast mr imaging: are signal intensity time course data useful for differential diagnosis of enhancing lesions. Radiology. 211, 101-110 (1999)

3. MD Schnall, S Rosten, S Englander, S Orel, L Nunes, A combined architectural and kinetic interpretation model for breast MR images. Acad. Radiol. 8, 591-597 (2001)

4. B Szabo, P Aspelin, M Wiberg, B Bone, Dynamic MR imaging of the breast - analysis of kinetic and morphologic diagnsotic criteria. Acta Radiologica. 44, 379-386 (2003)

5. S Agliozzo, MD Luca, C Bracco, A Vignati, V Giannini, L Martincich, A Bert, F Sardanelli, D Regge, Computer-aided diagnois for contrast-enhanced breast MRI of mass-like lesions using a multiparametric model combining a selection of morphological, kinetic and spatio-temporal features. Medi. Phys. 39, 3102-3109 (2012)

6. APS van der Velden, C Boetes, P Bult, T Wobbes, Variability in the description of morphologic and contrast enhancement characteristics of breast lesions on magnetic resonance imaging. Am. J. Surg. 192, 172-178 (2006)

7. G Grimsby, R Gray, A Dueck, S Carpenter, C Stucky, H Aspey, M Giurescu, B Pockaj, Is there concordance of invasive breast cancer pathologic tumor size with magnetic resonance imaging. Am. J. Surg. 198, 500-504 (2009)

8. IObdeijn, C Loo, A Rijnsburger, M Wasser, E Bergers, T Kok, J Klijn, C Boetes, Assessment of false-negative cases of breast MR imaging in women with a familial or genetic predisposition. Breast Cancer Res. Treat. 119, 399-407 (2010)

9. G Tourassi, R Vargas-Voracek, D Catarious, Computer-assisted detection of mammographic masses: a template matching scheme based on mutual information. Med. Phys. 30, 2123-2130 (2003)

10. G Tourassi, B Harrawood, S Singh, J Lo, Information-theoretic cad system in mammography: entropy-based indexing for computational efficiency and robust performance. Med. Phys. 34, 3193-3204 (2007)

11. G Tourassi, R Ike, S Singh, B Harrawood, Evaluating the effect of image preprocessing on an information-theoretic cad system in mammography. Acad. Radiol. 15, 626-634 (2008)
12. L Hadjiiski, B Sahiner, H Chan, Evaluating the effect of image preprocessing on an information-theoretic CAD system in mammography. Curr. Opin. Obstet. Gynecol. 18, 64-70 (2006)

13. M Kupinski, M Giger, Automated seeded lesion segmentation on digital mammograms. IEEE Trans. Med. Imaging. 17, 510-517 (1998)

14. E Rosen, S Smith-Foley, W DeMartini, P Eby, S Peacock, C Lehman, BI-RADS MRI enhancement characteristics of ductal carcinoma in situ. Breast J. 13, 545-550 (2007)

15. N Sakamoto, M Tozaki, K Higa, Y Tsunoda, T Ogawa, S Abe, S Ozaki, M Sakamoto, T Tsuruhara, N Kawano, T Suzuki, Yamashiro N, E Fukuma, Categorization of non-mass-like breast lesions detected by MRI. Breast Cancer. 15, 241-246 (2008)

16. H Yabuuchi, Y Matsuo, T Kamitani, T Setoguchi, T Okafuji, H Soeda, S Sakai, M Hatekenata, M Kubo, E Tokunaga, $\mathrm{H}$ Yamamoto, $\mathrm{H}$ Honda, Non-mass-like enhancement on contrast-enhanced breast MRI imaging: lesion characterization using combination of dynamic contrast-enhanced and diffusion-weighted mr images. Eur. J. Radiol. 75, 126-132 (2010)

17. TVag, P Baltzer, M Dietzel, R Zoubi, M Gajda, O Camara, W Kaiser, Kinetic analysis of lesions without mass effect on breast MRI using manual and computer-assisted methods of dynamic mr imaging features to the breast. Eur. Radiol. 21, 893-898 (2011)

18. R Lucht, S Delorme, J Heiss, M Knopp, MA Weber, J Griebel, G Brix, Classification of signal-time curves obtained by dynamic-magnetic resonance mammography. Invest. Radiol. 40, 442-447 (2005)

19. G Ertas, O Gulcur, O Osman, O Ucan, M Tunaci, M Dursun, Breast MR segmentation and lesion detection with cellular neural networks and 3D template matching. Comput. Biol.Med. 38, 116-126 (2008)

20. W Chen, M Giger, G Newstead, U Bick, Automatic identification and classification of characteristic kinetic curves of breast lesions on DCE-MRI. Med. Phys. 33, 2878-2887 (2006)

21. B Szabo, M Wilberg, B Bone, P Aspelin, Application of artificial neural networks to the analysis of dynamic MR imaging features to the breast. Eur. Radiol. 14, 1217-1225 (2004)

22. A Meyer-Bäse, T Schlossbauer, O Lange, A Wismüller, Small lesions evaluation based on unsupervised cluster analysis of signal-intensity time courses in dynamic breast MRI. Int. J. Biomed. Imaging (2010). doi: 10.1155/2009/326924

23. T Twellmann, A Meyer-Baese, O Lange, S Foo, T Nattkemper, Model-free visualization of suspicious lesions in breast MRI based on supervised and unsupervised learning. Eng. Appl. Artif. Intell. 21, 129-140 (2008)

24. T Schlossbauer, G Leinsinger, A Wismueller, O Lange, M Scherr, A Meyer-Baese, Classification of small contrast enhancing breast lesions in dynamic brmagnetic resonance imaging using a combination of morphological criteria and dynamic analysis based on unsupervised vector-quantization. Invest. Radiol. 43, 54-64 (2008)

25. D Newell, K Nie, J Chen, C Hsu, H Yu, O Nalcioglu, M Su, Selection of diagnostic features on breast MRI to differentiate between malignant and benign lesions using computer-aided diagnostics: differences in lesions presenting as mass and non-mass-like enhancement. Eur. Radiol. 20, 771-781 (2010)

26. SA Jansen, A Shimauchi, L Zak, X Fan, GS Karczmar, GM Newstaed, The diverse pathology and kinetics of mass, nonmass, and focus enhancement on mr imaging of the breast. J. Magn. Reson. Imaging. 33, 1382-1389 (2011)

27. S Jansen, Ductal carcinoma in situ: detection, diagnosis, and characterization with magnetic resonance imaging. Semin. Ultrasound, CT MRI. 32, 306-318 (2011)

28. JD Shutler, MS Nixon, Zernike velocity moments for sequence-based description of moving features. Image Vis. Comput. 24, 343-356 (2006)

29. S Behrens, H Laue, T Boehler, B Kuemmerlen, H Hahn, HO Peitgen, Computer assistance for MR based diagnosis of breast cancer: present and future challenges. Comput. Med. Imaging Graph. 31, 236-247 (2007)

30. T Brox, A Bruhn, N Papenberg, J Weickert, High accuracy optical flow estimation based on a theory for warping. Lecture Notes Comput. Sci. 3024, 26-36 (2006)

31. T Chan, L Vese, Active contours without edges. IEEE Trans. Image Process. 10, 266-277 (2001)

32. F Retter, Improved computer-aided diagnosis scheme for breast lesions in DCE-MRI based on motion artifact removal and integration of morphologic and dynamic information. Master's thesis, Dept. of Computer Science, Saarland University, Saarbrücken, Germany (2010) 
33. F Steinbrücker, Tumor classification on breast MR images. Master's thesis, Dept. of Computer Science, Saarland University, Saarbrücken, Germany (2008)

34. SH Lee, JH Kim, N Cho, JS Park, Z Yang, YS Jung, WK Moon, Multilevel analysis of spatiotemporal association features for differentiation of tumor enhancement patterns in breast DCE-MRI. Med. Phys. 37(8), 3940-3956 (2010)

35. SA Jansen, X Fan, GS Karczmar, H Abe, RA Schmidt, M Giger, GM Newstead, DCEMRI of breast lesions: is kinetic analysis equally effective for both mass and nonmass-like enhancement? Med. Physics. 35(7), 3102-3109 (2008)

36. JC Lagarias, JA Reeds, MH Wright, PE Wright, Convergence properties of the nelder-mead simplex method in low dimensions. SIAM. J. Optimization. 9, 112-147 (1998)

37. S Lespinats, A Meyer-Baese, F Steinbrücker, T Schlossbauer, Evaluation of visual exploratory analysis of DCE-MRI data of breast lesions based on morphological features and novel dimension reduction methods. Int. Conf. Neural Netw. 3, 1764-1770 (2009)

38. FB Fuller, The writhing number of a space curve. Proc. Natl. Acad. Sci. U.S. A. 68(4), 815-819 (1971)

39. A Lauric, E Miller, S Frisken, AM Malek, Automated detection of intracranial aneurysms based on parent vessel 3D analysis. Med. Image Anal. 14(2), 149-159 (2010)

40. A Lauric, E Miller, M Baharoglu, A Malek, 3D shape analysis of intracranial aneurysms using the writhe number as a discriminant for rupture. Ann. Biomed. Eng. 39, 1457-1469 (2011)

41. P Yap, R Paramesran, S Ong, Image analysis by krawtchouk moments. IEEE Trans. Image Process. 12, 1367-1377 (2003)

42. A Mademlis, A Axenopoulos, P Daras, D Tzovaras, M Strintzis, 3d content-based search based on 3d krawtchouk moments. Proc. Third. Int. Symp. 3D Data Process. Vis. Transm. 1, 743-749 (2006)

43. F Retter, C Plant, B Burgeth, T Schlossbauer, A Meyer-Baese, Improved computer-aided diagnosis for breast lesions detection in dce-mri based on image registration and integration of morphologic and dynamic characteristics. SPIE Symp. Comput. Intell. 8059, 80590J (2011)

44. MR Teague, Image analysis via the general theory of moments. J. Opt. Soc. Am. 70(8), 920-930 (1979)

45. M Aizerman, E Braverman, L Rozonoer, Theoretical foundations of the potential function method in pattern recognition learning. Automation Remote Control. 25, 821-837 (1964)

doi:10.1186/1687-6180-2013-172

Cite this article as: Hoffmann et al: Automated analysis of non-massenhancing lesions in breast MRI based on morphological, kinetic, and spatio-temporal moments and joint segmentation-motion compensation technique. EURASIP Journal on Advances in Signal Processing 2013 2013:172.

\section{Submit your manuscript to a SpringerOpen ${ }^{\mathcal{O}}$ journal and benefit from:}

- Convenient online submission

- Rigorous peer review

- Immediate publication on acceptance

- Open access: articles freely available online

- High visibility within the field

- Retaining the copyright to your article

Submit your next manuscript at $\boldsymbol{\wedge}$ springeropen.com 